\title{
Soups \& SELEX for the origin of life
}

\section{RENÉE SCHROEDER}

Max F. Perutz Laboratories, University of Vienna, 1030 Vienna, Austria
The theory of evolution quickly brought up the most fascinating question in biology: the origin of life. How did life emerge? The biggest challenge in this endeavor to understand how life could have emerged without a controller is to unravel the chemical processes that made the synthesis of complex molecules containing information possible.

Already at high school I was totally caught by Oparin's hypothesis that simple unicellular organisms evolved from simple organic molecules, which in turn originated from simple inorganic molecules present in the early atmosphere of our planet. Oparin and Haldane coined the idea of "primordial soup" experiments. For my "Matura" (that is the name of the exam you do in Austria, when you finally leave high school) I got Stanley Miller's famous primordial soup experiments as question. I just now realize that ever since SOUP \& SELEX experiments are the ones I find most seminal for shaping our view of the world. I still consider these experiments as the breakthrough in biology of the 20th century because it opened up a whole new field clearly suggesting that one day it might be possible to understand how life emerged.

Until the discovery of microorganisms by Louis Pasteur, fermentation by microorganisms was thought to be the product of spontaneous generation. Until Eduard Buchner demonstrated that fermentation could occur outside a living organism, in a test tube, it was the belief that only living cells were able to catalyze biochemical reactions. When in 1897 Bucher reported that a cell-free extract from yeast was able to ferment sugar, the field of biochemistry was invented and the analysis of chemical reactions taking place in living cells became accessible.

There have been several theoretical approaches to the emergence of life as a self-replicating system, whereby the most essential part of all these concepts is the idea that complex systems can evolve from simple compounds without an external controller. This was indeed a revolutionary thought! As Erwin Schrödinger pointed out in 1944, it looks like living organisms bypass the second law of thermodynamics because they are able to evolve to complex systems out of disordered compounds ignoring Maxwell's Demon. Manfred Eigen, Leslie Orel and Stuart Kauffmann coined theoretical back-

Corresponding author: renee.schroeder@univie.ac.at

Article and publication date are at http://www.rnajournal.org/cgi/doi/10. 1261/rna.050559.115. Freely available online through the RNA Open Access option. grounds on which chemists could develop experimental set ups. Combinatorial events of simple chemical reactions could lead to stably replicating populations of increasing complexity.

\section{Primordial Soups: prebiotic chemistry needs to deliver activated ribonucleotides}

One prerequisite for the emergence of Life is the condensation of informational polymers by purely chemical means. Indeed, the Orgel lab has been successful in obtaining nucleotide polymers as long as 55 monomers in the presence of mineral surfaces like montmorillonite. For this to happen activated ribonucleotide building blocks must have been available in sufficient amounts. This essential aspect of the hypothesis has been proven especially difficult because the addition of nucleobases to ribose is inefficient for purines and does not occur for pyrimidines.

Performing Stanley Miller-type soup experiments is one useful approach and determining the content of meteorites and interstellar gases is another fascinating way to learn about geo- and prebiotic chemistry. Recently Patrick Thaddeus reported the finding of over 130 molecules in interstellar gas and circumstellar shells with quite impressive molecular weights of up to 147 Daltons. Among these are compounds, which have been shown to condensate to pyrimidines like cyanamide, cyanoacetylene, glycolaldehyde, glyceraldehyde and most importantly inorganic phosphate. The Sutherland laboratory has proven that starting from these compounds one can bypass the difficult condensation of ribose with nucleobases by using alternative routes. Reaction of cyanamide and glycolaldehyde results in the heterocycle 2-amino oxazole, which adds to glyceraldehyde to give pentose amino-oxazolines. Just 2 more steps are necessary to obtain $B$ ribocytidine- $2^{\prime}, 3^{\prime}$-cyclic phosphate: the reaction of arabino amino-oxazoline with cyanoacetylene results in the anhydroarabinonucleoside which then undergoes phosphorylation. There are growing arguments in favor of $2^{\prime}-3^{\prime}$ cyclic phosphates instead of triphosphates being a probable form of activated nucleotides for their condensation to form the first RNA oligomers.

(C) 2015 Schroeder This article, published in RNA, is available under a Creative Commons License (Attribution-NonCommercial 4.0 International), as described at http://creativecommons.org/licenses/by-nc/4.0/. 


\section{Reconstructing the RNA World, the molecular biologist's dream}

In 1966, Norm Pace and Sol Spiegelman reported that RNA could serve as template for the synthesis of RNA! RNA could thus be the "instructive agent in a replicative process" and hence "satisfy the operational definition of a self-duplicating entity". Furthermore, this work provided a set-up for studying the genetics and evolution of a self-duplicating nucleic acid molecule. With this landmark discovery and technical setup it became clear that studying the origin of a self-replicating instructive system was amenable to empirical enquiry. As a consequence of this observation and many other discoveries on the properties of nucleic acids, Carl Woese, Francis Crick and Leslie Orgel suggested that RNA was the first informative molecule. The most exciting discovery on the road to an RNA World theory were the catalytic properties of RNA by the laboratories of Thomas Cech and Sidney Altmann. After this discovery, the idea of an "RNA World" was at hand.

Once this RNA World hypothesis was established, it became evident that we needed an experimental proof of concept. The challenge for pursuing experiments on the evolution of a self-replicating informational system was a question of getting all necessary tools to do so. In 1970 Howard Temin and David Baltimore discovered reverse transcriptase, the enzyme that can make DNA from an RNA template. This was an essential discovery for the RNA World theory because it requires that DNA came later than RNA and it is necessary for the concept of an RNA world. Furthermore, the purification of reverse transcriptases allowing to synthesize DNA from an RNA template in vitro, was an essential tool for the development of in vitro evolution techniques. Just a few more tools were necessary to start experiments on the in vitro evolution of RNA: random synthesis of oligonucleotides and the establishment of the polymerase chain reaction making it possible to specifically amplify DNA sequences of choice. By the end of the ' 80 s and beginning of the ' 90 s, the way was paved for the invention of an ingeniously efficient way to amplify, mutate, and select RNA molecules with desired properties: SELEX, the Systematic evolution of ligands by exponential enrichment. We were ready to start playing the game of evolution!

\section{SELEX - in vitro evolution of RNA}

The selection of a high affinity binding nucleic acid sequence from a pool of random sequences was first reported from the laboratory of Kevin Struhl for the determination of the specific DNA sequences recognized by the yeast transcription factor GCN4. Shortly after, in 1990, RNA sequences that bind with high affinity to T4 phage DNA polymerase were isolated and the SELEX technology established by Craig Tuerk and Larry Gold. In this procedure reiterate cycles of ligand selection from RNA pools with a very high number of variants and amplification of the selected sequences lead to the enrichment of high affinity ligands. Multiple rounds lead to an ex- ponential accumulation of a population of sequences that best match the conditions set for the selection step.

\section{In vitro selection of RNA aptamers that bind small ligands}

In 1990 Andy Ellington and Jack Szostak performed the first set of SELEX experiments aiming at the isolation of RNAs that bind small molecules with high affinity and specificity. These RNAs were termed "aptamers." The goal of these experiments was to explore how RNAs can fold into threedimensional structures to form binding pockets for small molecules and to show that RNAs can bind almost all types of small molecules and proteins. The only example of an unsuccessful SELEX experiment I heard of was the isolation of aptamers against testosterone; this is probably due to the low solubility of testosterone in water to initiate binding to RNA. Aptamers were selected against organic dyes, nucleotides, cofactors, amino acids and antibiotics. It was for example impressive to realize that RNA aptamers could discriminate 10,000-fold between small molecules as similar as theophylline and caffeine.

\section{In vitro selection of RNA aptamers that bind proteins-RNA Therapeutics}

Very soon it was realized that SELEX represented an exceptional tool to isolate therapeutic aptamers targeting specific proteins. Craig Tuerk, McDougal and Larry Gold reported the first example in 1992. They selected aptamers against HIV-1 reverse transcriptase and obtained RNA pseudoknots that were not only able to bind to the protein, but also inhibit its activity. It became obvious that having SELEX in hand to isolate RNA aptamers that target proteins was an unprecedented and versatile tool for the development of diagnostics and therapeutics. There is a very long list of aptamers targeting proteins that cause diseases. The major drawbacks for this strategy are the low stability of RNA and the delivery to the desired site of action. Many efforts have been undertaken to bypass these limitations.

Craig Tuerk and Larry Gold's observation that RNA aptamers could often inhibit the targeted protein was the start of a long effort to obtain RNA therapeutics. In 2004 the US Food and Drug Administration (FDA) approved the first RNA aptamer based therapeutic: Macugen or Pegaptanib. It is a chemically modified 28-mer aptamer that interferes with the vascular endothelial growth factor VEGF reducing the development of age-related macular degeneration.

\section{In vitro evolution of RNA enzymes}

The essential question for the survival of the RNA World theory is very demanding: can RNA catalyze all reactions necessary for self-replication on early Earth? To answer these questions an RNA world needed to be reconstructed de novo. The Szostak, Bartel and Joyce labs started with this 
truly heroic enterprise. The number of reactions required to sustain a system as complex as Life is intimidating. In addition, the number of possible conditions is even more intimidating. Nevertheless, SELEX was used to evolve a large number of essential catalytic activities like RNA polymerization, RNA ligases, nucleotide synthesis, polynucleotide kinases to mention just the most prominent. These experiments are much more demanding on the selection strategies, because catalysts do not bind tightly to their substrates or products long enough to survive the selection procedures. New strategies had to be invented.

The first attempts in the Joyce lab used the Tetrahymena group I intron ribozyme and evolved the intron RNA in such a way that it would perform a different reaction by using different substrates. In 1992 Beaudry and Joyce reported that they succeeded in obtaining an RNA enzyme that cleaves DNA in a sequence specific manner: out of a population of $10^{13}$ variants of the Tetrahymena ribozyme they enhanced the DNA cleavage activity 100 -fold.

\section{The first informative replicator}

The emergence of a self-replicating RNA, that catalyzes its own replication is highly improbable, because it would have to unfold to be copied and thereby loose its activity. From everything we know about the characteristics of RNA, it needs to fold into defined three-dimensional structures to exert catalytic activity. In addition, by copying itself, it would produce its complement resulting in a double stranded RNA. Therefore, what needed to pop up was more than one copy of an RNA that could accelerate the RNA-templated condensation of activated nucleotides in trans.

There are several scenarios how this first replicator might have evolved; several steps are necessary to get started. First activated nucleotides need to condense to form short chains. It is highly probable that this condensation took place on some kind of template, inorganic or organic, maybe even short peptides (see below). This would increase the appearance of similar molecules. Once such short chains pop up, their abundance can be increased by unanalyzed RNA-templated copying. Indeed, the Szostak lab demonstrated that it is possible to synthesize the condensation of RNAs without chemical catalysis using RNA as template. The problem with this procedure is that the results are double-stranded RNAs, which need to be melted. A further solution for this problem is temperature cycling during night and day or cold and warm sections that might help dissociate these RNAs.

Next, we need compartments to avoid diffusion and dilution of these first active molecules. Packaging these primordial RNAs into lipid vesicles provides a path to a first kind of cell. The Szostak lab has made tremendous progress in recreating this first prebiotic protocell. By carefully choosing the right buffer conditions and concentration of $\mathrm{Mg}^{2+}$ ions, they showed that RNAs could replicate inside fatty acid vesicles. By adding citrate to this soup, they could avoid the dis- ruptive effect of $\mathrm{Mg}^{2+}$ on the fatty acid membranes and protect the single stranded RNAs from hydrolysis.

This can already be viewed as a successful simple scenario for the emergence of a simple form of RNA-based life.

A question that I like asking young students and lay public is what would have happened if the very first RNA replicase had been perfect not allowing mistakes or variations? Normally, I get first thinking faces and very quickly I get the right answer: There would have been no evolution and a single type of RNA molecule might have covered the earth. Thinking through this Gedankenexperiment, one immediately understands the major principle of evolution.

\section{RNA World of RNP World?}

Many scenarios have been discussed for the origin of life, mainly based on the assumption that in addition to many small metabolites, amino acids, nucleic acids and lipids were the main players. The RNA world hypothesis is therefore not an RNA only hypothesis and we should not forget RNA's biggest friends, the proteins. Here I want to argue for a co-evolution of RNA and short peptides from very early on. Until today there are many highly active short peptides, which are not synthesized on ribosomes but on non-ribosomal peptide synthetases. These products of secondary metabolism might provide hints to what kind of peptides could have been synthesized without the need of ribosomes and what kind of synthesis was available under primordial conditions. When taking a look at this enormous reservoir of interesting compounds one might find small peptides, which have interesting effects on RNA. I want to mention just one fascinating small molecule: viomycin. Viomycin is a small cyclic peptide from the tuberactinomycin family of antibiotics, which inhibits bacterial protein synthesis and several ribozymes. But most importantly, my lab found that viomycin is not only an inhibitor but also an activator of ribozymes at sub-inhibitory concentrations. It interacts with group I intron RNA leading to the formation of intron oligomers thus inducing RNARNA interactions and RNA-RNA recombination. Because viomycin interacts with many pseudoknot-containing RNAs altering their activity, it is a good example for a model peptide that could have served as mould for the very early condensation and replication of RNA molecules. Julian Davies has always stressed the notion that antibiotics were not merely invented to kill life, but that these compounds are effector molecules that modulate a large number of activities.

\section{Self-regulation}

In addition to being informative and catalytic, the last 20 years demonstrated the capacity of RNA as a regulatory molecule. A myriad of micro- and macroRNAs control gene expression and thereby shape the development of organisms.

There is one very important aspect to take into account when studying complex systems: They regulate themselves! 
Therefore the type of RNA I am most interested in at the moment are RNAs that can regulate their own synthesis.

The reconstruction of the RNA world is a project that will never be done, because like evolution it will continue to evolve as long as somebody will be working on it. Whatever its outcome will be, it is clear that these projects revolutionized our approach to science in general and our view of the world in particular.

\section{Acknowledgments}

I want to thank the RNA community for being a home to so many of us working on different aspects of RNA and for creating an environment of enthusiasm and friendship. You made all these wonderful insights into Life possible.

Research in my laboratory is funded by the Austrian Funding Agency FWF, the European Union and the Austrian Ministry of Science. 

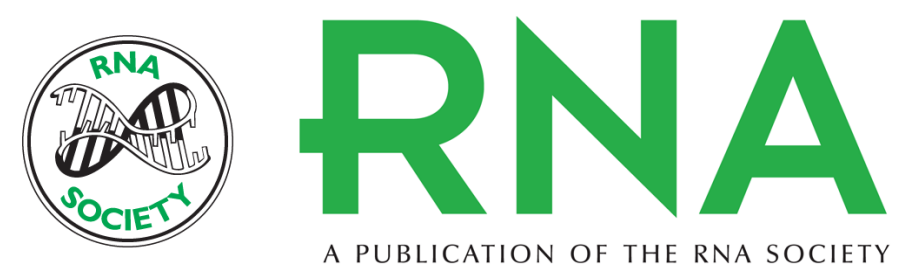

A PUBLICATION OF THE RNA SOCIETY

\section{Soups \& SELEX for the origin of life}

Renée Schroeder

RNA 2015 21: 729-732

Open Access Freely available online through the RNA Open Access option.

Creative This article, published in $R N A$, is available under a Creative Commons License

Commons (Attribution-NonCommercial 4.0 International), as described at

License http://creativecommons.org/licenses/by-nc/4.0/.

Email Alerting Receive free email alerts when new articles cite this article - sign up in the box at the Service top right corner of the article or click here. 